Cahiers de recherches médiévales

Journal of medieval studies

$1 \mid 1996$

Croisades et idée de croisade à la fin du Moyen Âge

\title{
Guillaume-Sanche, Elie de Pommiers et leurs frères
}

vers $1340-1360$

Françoise Beriac et Eric Ruault

\section{CpenEdition}

Journals

Édition électronique

URL : https://journals.openedition.org/crm/2534

DOI : $10.4000 / \mathrm{crm} .2534$

ISSN : 1955-2424

Éditeur

Honoré Champion

Édition imprimée

Date de publication : 15 janvier 1996

Pagination : 207-227

ISSN : $1272-9752$

Référence électronique

Françoise Beriac et Eric Ruault, « Guillaume-Sanche, Elie de Pommiers et leurs frères », Cahiers de recherches médiévales [En ligne], 1 | 1996, mis en ligne le 07 février 2008, consulté le 15 décembre 2022. URL : http://journals.openedition.org/crm/2534; DOI : https://doi.org/10.4000/crm.2534 


\section{Guillaume-Sanche, Elie de Pommiers et leurs frères vers 1340-1360}

La première partie d'un article paru en 1975, «Les bases sociales du pouvoir anglogascon au milieu du XIV ${ }^{\mathrm{e}}$ siècle ${ }^{1}$, reprenait les pages que $P$. Capra consacrait à la noblesse gasconne dans sa thèse soutenue trois ans plus tôt, L'administration anglogasconne au temps de la lieutenance du Prince Noir (1354-1362). ${ }^{2}$ Aux hasards de deux notes $^{3}$, on voit que l'auteur avait en projet deux articles sur des figures marquantes: Guillaume-Sanche de Pommiers et son frère cadet Elie, l'un comme chef de file des nobles du Bordelais et du Bazadais, l'autre pour le caractère remarquable de sa carrière de chef militaire toujours sur la brèche, et devant tout ou presque à la faveur du prince. En 1979, paraissait celui sur Elie de Pommiers ${ }^{4}$, P. Capra ne put donner suite au reste de ses projets. Son dessein premier n'était assurément ni une biographie, ni une monographie familiale; «Les bases sociales du pouvoir anglo-gascon» se concluait sur une approche globale de l'attitude politique de la noblesse gasconne : les plus actifs partisans du roi-duc, ceux qui s'engageaient à fond dans toutes les occasions militaires de la défense du duché, puis des chevauchées princières, n'étant que quelques activistes notoires, «un groupe de 25 capitaines toujours disponibles» 5 . Guillaume Sanche et Elie étaient de ceux-là. P.Capra les avaient retenus comme exemplaires et significatifs d'un groupe nobiliaire.

Fin 1993, les hasards d'un travail sur la trahison de Guillaume-Sanche IV 6 de Pommiers ont amené à reconsidérer le cas de son père et homonyme et de son oncle Elie, en consultant les travaux de P. Capra, ce qui était édité, mais aussi le reste de sa thèse; l'extrême richesse du dossier sur les frères Pommiers a abouti à cette exposition parallèle de leurs carrières, qui pour l'essentiel se base sur les travaux de P. Capra, en rassemblant tout ce qui concernait Elie et Guillaume-Sanche, au fil de sa thèse. Depuis, un mémoire de

\footnotetext{
1 «Les bases sociales du pouvoir anglo-gascon au milieu du XIVe siècle», Le Moyen Age, $1975, n^{\circ}$ 2, LXXXI, pp. 273-299 (=Capra, Les bases sociales du pouvoir anglo-gascon ).

${ }^{2}$ Paris IV, 1972, 3 vol. dactyl.

${ }^{3}$ Les bases sociales du pouvoir anglo-gascon, n. 127 (p. 292) et 133 (p. 294).

${ }^{4} \mathrm{P}$. Capra, «Une carrière anglo-gasconne au milieu du XIV siècle, Elie de Pommiers», Société et groupes sociaux en Aquitaine, Bordeaux, 1979, pp. 57-62. (=Capra, Une carrière anglo-gasconne au milieu du XIV siècle).

5 Les bases sociales du pouvoir anglo-gascon, p. 293, 299.

${ }^{6} \mathrm{~F}$. Bériac, Trahison, fidélité ou attentisme, les attitudes de la noblesse gasconne en 1377, à paraître dans les Actes du colloque du CRISIMA (Montpellier, novembre 1995) Félonie, trahison, reniements au Moyen Age.
}

Cahiers de Recherches Médiévales (XIIf -XV s.), 1, 1996 
maîtrise avait permis d'avoir une vue d'ensemble de la famille de Pommiers du XIII au $\mathrm{XV}^{\mathrm{e}}$ siècle, à partir d'une recension des sources publiées?

L'heure était venue d'une mise en forme de ce dossier, en tenant compte des publications, finalement abondantes, qui ont renouvelé nos connaissances sur le rôle politique et militaire de la noblesse à la fin du Moyen $\mathrm{Age}^{8}$, et qui ne pouvaient entrer dans les vues de $P$. Capra, dont les travaux sur ce thème paraissent en 1972/1975. Sa mort est survenue alors que notre article n'était qu'un projet, l'indispensable dialogue entre coauteurs n'a pu avoir lieu, mais nous nous sommes efforcés de suivre la pensée et les mots de P. Capra. Pour l'essentiel, il s'agit bien de l'utilisation de son travail, dans une présentation et dans une perspective qui n'étaient pas celles de l'ensemble de sa thèse, mais qui se veulent un hommage à son œuvre.

Vers 1335 à la mort de son père et homonyme, Guillaume-Sanche III de Pommiers hérite du domaine de Pommiers près de La Réole; la localité de Saint-Pierre de Pommiers porte encore le nom de cette seigneurie somme toute assez modeste. Les sires de Pommiers possèdent des biens assez respectables groupés en deux blocs : l'un vers Civrac-Gensac au nord, l'autre dans les environs de leur château éponyme. A l'époque des Recognitiones feudorum, soixante ans plus tôt, ces possessions s'éparpillaient entre Dordogne et Garonne sur vingt-quatre localités, et ils n'avaient alors que quatre vassaux. Cette famille n'avait que médiocrement proliféré et l'habitude de garder les biens de l'héritage en co-seigneurie avait limité le développement de branches cadettes et empêché l'émiettement de leur fortune foncière et banale. Depuis le début du XIV ${ }^{e}$ siècle, les Pommiers se sont mis au service du roi-duc, mais cette famille ne compte pas vraiment de personnages de grand relief, ni parmi les laïques ni parmi les quelques cadets à avoir fait de petites carrières ecclésiastiques. Le sire de Pommiers se rattache à la zone des confins bordelais, bazadais et agenais, où un nombre particulièrement grand de ses pareils sert activement la cause anglo-gasconne. Guillaume-Sanche III est le chef d'un nombreux lignage, puisqu'il a au moins quatre frères, Elie, Amanieu, Bertrand et Jean ${ }^{9}$, peut-être cinq si l'on compte parmi eu un Arnaud de Pommiers, capitaine de La Réole en $1373^{10}$.

P. Capra voit en lui un de ces vassaux qui forment le second rang, mais d'une façon encore notable, de la noblesse guyennoise. R. Boutruche, prenant en compte la famille de Pommiers globalement aux XIII ${ }^{e}-X{ }^{e}$ siècles, la situe de façon plus optimiste parmi les «grands seigneurs» avec les Montferrand, Lesparre, Fronsac, Budos, Noaillan, La Trau; il relève le caractère "encore imposant de la forteresse de Pommiers" et note que, comme tous «les gentilshommes de haut rang», les Pommiers ont une demeure à Bordeaux, dans la

\footnotetext{
${ }^{7}$ E. Ruault, La famille de Pommiers au Moyen Age, TER 1987, Bordeaux III sous la dir. de B. Guillemain.

${ }^{8} \mathrm{Cf}$. le récent ouvrage de M.-T. Caron, Noblesse et pouvoir royal en France, XIII $-X V I^{e}$ siècle, Paris, 1994, avec une abondante bibliographie.

${ }^{9} \mathrm{Cf}$. un mandement autorisant divers seigneurs à se faire représenter en justice, PRO C61/70 mbr 1, 16, 17, 6 juin 1358 (désormais nous ne préciserons pas «PRO», toutes les sources manuscrites utilisées en provenant.

${ }^{10}$ Arch. hist. de la Gironde, t. XIII, p. 331, 335.
} 
vieille ville, entre les rues de la Petite-Carpenteyre et de la Devise ${ }^{11}$. Eux et leurs pairs mènent un genre de vie à la fois militaire et terrien imposé par leur fortune essentiellement seigneuriale et «le service du fief, la tradition et les circonstances.» ${ }^{12}$ Notre objet n'était pas ici de trancher entre deux appréciations de la fortune des Pommiers vers 1340, mais assurément, le fait que ce soit Guillaume-Sanche III et non son fils comme le supposait P. Capra, qui ait épousé l'héritière de la vicomté de Fronsac ${ }^{13}$, fait pencher la balance en faveur d'une situation familiale solide.

Guillaume-Sanche, aîné d'une belle brochette de garçons ayant survécu à l'enfance, a emporté la «seigneurie mère» mais ses cadets avaient le droit à leur «soutenance», dans un triste contexte a priori puisqu'Edouard III, puis le Prince Noir donneraient moins de pensions et de places aux Gascons que leurs prédécesseurs. ${ }^{14}$ Outre que cette dernière appréciation ne semble pas valoir en ce qui concerne au moins le Prince ${ }^{15}$, elle laissait de côté le contexte guerrier qui plaçait les nobles gascons devant de nouvelles occasions, que les frères Pommiers surent très bien saisir. Cette génération de frères ne suit pas les mêmes voies que les précédentes où l'on observait un aîné entouré de cadets ou de cousins coseigneurs vivotant dans son ombre, ou flanqué de parents médiocrement casés dans des bénéfices ecclésiastiques; cette remise en cause de l'équilibre familial traditionnel porte la fratrie à son apogée.

\section{$1339 / 1340$ : de jeunes seigneurs gascons se tournent vers la guerre}

\section{Les débuts d'un jeune baron}

Guillaume-Sanche III, né au plus tard vers 1315/1320, fait figure d'homme important dont l'adhésion compte, dès après le décès de son père vers 1335 ; il est des vassaux gascons qui reçoivent une lettre d'Edouard III, pour solliciter leur fidélité; ses terres essuient le premier choc des raids français en 1340-1341 et il se retrouve vite capitaine de La Réole. ${ }^{16}$

Son attitude procède d'un choix personnel ; Pierre, un de ses cousins, se tourna du côté français et Guillaume-Sanche recueillit en 1341 ses biens confisqués par Edouard III ; peut-être au même moment, en tout cas avant 1354, il capta ausi les dépouilles d'un autre rebelle Gaillard de Preissac, mais les céda à son frère Jean qui eut du mal à en profiter, au

\footnotetext{
11 Arch. hist. de la Gironde, t. XXI, p. 285 (comptes de l'archevêque).

${ }^{12}$ La crise d'une société. Seigneurs et paysans du Bordelais pendant la guerre de Cent Ans, Paris, rééd. 1963, p. 79, 81 et n. 2, 167 (=Boutruche, La crise d'une société). Voir aussi S. Faravel, La seigneurie, le château et la «ville» de Pommiers, Cah. du Bazadais, n 93, 1991, pp. 27-31.

13 Ruault, La famille de Pommiers, pp. 87-89. Il reste toutefois un point très obscur: quand Guillaume-Sanche III a réellement pris possession de la vicomté de sa femme.

14 Boutruche, La crise d'une société , 350-351.

15 C. Meyran, Nobles et officiers dans la Gascogne de Richard II, TER Bordeaux III, 1994, II, annexe III, fiefs de bourse et pensions (remontant pour une bonne part à 1356/1370), pp. 22-23. Cf aussi Register of Edward the Black Prince, Londres, 1930-1933, vol II-IV.

16 T. Rymer, Fodera, conventiones, litteræ inter reges Angliæ et alios, La Haye, 1739-1745. II, part. III, p. 172. T. Carte, Catalogue des rolles gascons, normans et françois..., LondresParis, 1743, p. 106.
} 
gré des hésitations de Gaillard I et Gaillard II entre France et Angleterre ${ }^{17}$. Les revenus de Guillaume-Sanche s'arrondirent aussi à partir de 1342 de toute une série de rentes données par le roi-duc: la coutume de Royan concédée par mandement du $1^{\text {er }}$ juin 1342 revient plusieurs fois dans les comptes de Stretley. ${ }^{18}$

A ce moment, Guillaume-Sanche a quitté ses horizons familiers pour guerroyer en Saintonge, car il prend lui-même le château de Blanzac aux Français et le garde à ses frais pendant seize ans avant d'en recevoir concession en $1358 .{ }^{19}$ Durant ces années, GuillaumeSanche participe à l'expédition du comte de Derby qui reprend Bergerac; il est d'ailleurs fait chevalier le jour même (24 août 1344), si l'on en croit la Petite Chronique de Guyenne. ${ }^{20}$ On ne peut reconstituer en détail les faits et gestes de Guillaume-Sanche dans le sillage du comte de Derby, en Agenais (1345) et en Saintonge à nouveau (1346) ; en tout cas, l'homme accède à une sorte de notoriété militaire flatteuse qui va jusqu'en France, puisque la Chronique normande cite, de façon un peu fantaisiste, les aveux d'un personnage qui aurait été un pillard de la compagnie du sire de Pommiers. ${ }^{21}$ La chancellerie pontificale aussi le connaît sous cet aspect! Elle lui envoie une missive sévère pour lui intimer l'ordre de libérer l'abbé de la Couronne, et de rendre les biens de l'abbaye dont certains «fils de Bélial» se sont emparés en son nom; trois mois plus tard, le sire de Pommiers qui s'est exécuté, a droit à des remerciement aimables, et on apprend à l'occasion que la curie avait fait des démarches à sa demande pour obtenir la libération d'un chevalier bordelais, Amanieu Colom, qui s'est du reste échappé avant même l'arrivée de la lettre pontificale. Cet homme de poids fait aussi partie des vingt-sept grands personnages du Sud-Ouest, tant côté français que côté anglais, auxquels le pape adresse des lettres d'introduction pour ses nonces chargés d'une mission de paix en décembre 1349.22 Guillaume-Sanche jouit donc, à la faveur du début de la guere, d'une notoriété de baron et de prédateur qui dépasse les horizons étroits où se confinait la réputation de ses ancêtres.

Son seigneur ne l'oublie pas qui lui donne aussi une bonne partie des revenus de la prévôté de La Réole, sans du reste qu'il rende compte du surplus. ${ }^{23}$ Guillaume-Sanche la garde jusqu'en $1357^{24}$; il a eu soin d'obtenir une rente ducale à percevoir près de ses domaines patrimoniaux, cela ne pouvait que contribuer à son prestige local. GuillaumeSanche s'est donc orienté très tôt vers le service actif de son seigneur, il ne se contentait pas d'administrer sa fortune et de louvoyer entre les deux rois, il avait clairement choisi son camp, n'en varia pas, et commença assez vite à engranger les modestes bénéfices de son engagement. Sa fidélité et ses intérêts allaient de pair. Qu'en est-il de ses frères cadets, Elie, Jean, Amanieu, Bertrand et Arnaud ? ${ }^{25}$

17 Carte, Catalogue des rolles gascons, p. 106, 140 ; C61/66 mbr 16, C61/70 mbr 17.

$18 \mathrm{E} 372 / 207$ fol.14r mbr 1 , fol. $15 \mathrm{r}$ mbr 1 et fol. $16 \mathrm{v}$ mbr 1 .

19 C61/70 mbr 14, 15/03/1358.

20 Archives municipales de Bordeaux, V, p. 400.

21 Chronique normande du XIV siècle, éd. A. et E. Molinier, Paris, 1882, p. 226.

22 Clément VI. Lettres se rapportant à la France, éd. E. Déprez et G. Mollat, t. III, 1959, n $4339,4340,4794,4930$.

${ }^{23}$ L'administration anglo-gasconne, p.118 n. 11 (réf. incomplète) dès 1349/50 au moins cf p. 391 n. 23, E372/207 fol. $15 \mathrm{r} \mathrm{mbr} 1$.

24 C61/69 mbr 13.

25 Ruault, La famille de Pommiers, p. 157, arbre généalogique. L'ordre de naissance des cadets n'est pas connu. 


\section{Des cadets aux armées}

Tous ont été des capitaines au service du roi-duc, au moins à un moment de leur vie, par exemple Bertrand en 1354 avec dix hommes d'armes. ${ }^{26}$ Nous ne connaissons bien que les «débuts» d'Elie, qui suivent de peu ceux de son ainé ; un cadet devait impérativement se faire une situation autonome au plus vite. Elie hérite quelques droits, sans plus ${ }^{27}$, la voie qui s'ouvrait à ce puiné n'était pas a priori celle de la fortune! Il a fallu pourvoir très tôt à son établissement. Nous le voyons déjà chanoine de Bazas au moment de sa nomination comme conseiller ducal par Olivier de Ingham, le 10 décembre 1336; ce jeune clerc bien né commence là parmi d'autres clercs une carrière que chacun devait voir d'Eglise. Notre homme est toujours chanoine en $1342 / 1344$, et probablement guère au-delà. ${ }^{28}$

Sa carrière ecclésiastique a vite tourné court, son engagement pour la cause de son seigneur ne pouvait guère plaire au pape ; Edouard III en mars 1344 lui promet un bénéfice vacant en Angleterre d'un revenu de $60 \mathrm{l}$. au plus, puis une rente compensatoire ${ }^{29}$, mais Elie change de voie, il se tourne vers les armes. Cette reconversion s'opère alors qu'il est certainement encore assez jeune, il avait pu avoir toute dispense de ne pas recevoir les ordres majeurs pendant quelques années; en tout cas, cela indique que son éducation n'avait pas été seulement cléricale et que le second(?) fils vivant du sire de Pommiers avait eu la jeunesse ordinaire d'un noble, avec chasse et exercices martiaux.

Son choix procède peut-être d'un goût personnel, mais certainement aussi des circonstances. Dix ou quinze ans auparavant, la protection d'un cardinal originaire de la Gascogne aurait offert à ce cadet besogneux de souriantes perspectives ecclésiastiques, espérer une mitre n'aurait rien eu de déraisonnable ; Bernard, un des oncles d'Elie avait été un familier de Clément $\mathrm{V}$, mais cela remontait à une trentaine d'années. ${ }^{30}$ Un des seuls seigneurs gascons à pouvoir désormais se flatter de très bonnes entrées personnelles à Avignon est Florimond de Lesparre, neveu du cardinal de Périgord ${ }^{31}$, mais encore enfant à cette date, et ce fils héritier n'eut pas besoin de chercher fortune dans l'Eglise. Le dossier des possibilités de carrières pour clercs gascons au-delà de 1330-1340, par le biais de protections avignonnaises, mériterait toutefois un examen systématique, car l'index des Lettres communes d'Urbain $V$ indique bien qu'ils grapillent quelques miettes du grand festin des clercs méridionaux. En ce qui concerne la famille de Pommiers, on repère Arnaud, chanoine de Saint-Emilion qui a bénéficié de la protection du cardinal Audouin Aubert, parent d'Innocent VI, pour un modeste cumul de bénéfices ${ }^{32}$. Il n'en est pas moins

\footnotetext{
${ }^{26} \mathrm{E} 101 / 169 \mathrm{n}^{\circ} 1$.

27 Procès pour les défendre, en février $1359, \mathrm{C} 61 / 72 \mathrm{mbr} 9$.

28 Capra, Une carrière anglo-gasconne, p. 58.

29 Calendar of Patent Rolls, 23/06/1340, 22-24/03/1344.

30 Ruault, La famille de Pommiers pp. 33-34, 157 ; voir aussi N. de Peña, «Vassaux gascons au service du roi d'Angleterre dans la première moitié du XIVe siècle : fidélité ou esprit de profit ?», Ann. du Midi, , t. 88, n 126, 1976(=Peña, Vassaux gascons au service du roi d'Angleterre ), p. $11 .$.

${ }^{31}$ Meyran, Nobles et officiers, I, p. 103.

$32+$ Lettres communes, $n^{\circ} 3518$ (18 novembre 1362); il est plus malaisé de formuler un jugement sur un autre Amaud, clerc du diocèse de Condom non constitué dans les ordres $\left(\mathbf{n}^{\circ}\right.$ $12127)$, et sur Pierre $\left(n^{\circ} 15567\right)$, Jean $\left(n^{\circ} 2577\right)$ et Gérard $\left(n^{\circ} 26009\right)$ qui ont eu des carrières
} 
clair que la manne avignonnaise n'allait pas en priorité aux sujets gascons du roi d'Angleterre.

D’autre part, ce dernier pouvait difficilement se permettre de caser sur des bénéfices anglais tous les clercs gascons déçus par le peu de faveur où ils étaient désormais à la curie ; le destin de son oncle Pons, mort en 1341 chanoine de Saint-André de Bordeaux, ou celui de son oncle Jean, prieur d'Andover, établissement du diocèse de Winchester, jusqu'en 1358, ne tentèrent pas Elie, et il faut dire que vers 1340 , l'affaire se présentait fort mal. La conjonction de la faveur royale et de la bienveillance pontificale, vers 1330 , permettait à des clercs gascons de disposer d'appétissantes prébendes en Angleterre, rien que pour l'année 1331, on relève le jeune Pons de Pommiers à York, Ithier de Concoret à Hereford, Raimond Pélegrin à Wells, Pierre de Galicien à Canterbury et Isarn Jordan à Salisbury. De son côté, Jean de Pommiers avait reçu licence en décembre 1330, d'aller faire des études de théologie ou de droit canonique dans un studium de son choix, autrement dit de ne pas résider à Andover. ${ }^{33}$ Mais quand Elie arrive à l'âge adulte, les débuts de la guerre de Cent ans jettent les clercs gascons détenteurs de bénéfices anglais dans l'embartas: ils ont bien du mal à échapper à la saisie des bénéfices tenus par gens du royaume de France. Jean de Pommiers remontre bien qu'il est natif d'Aquitaine et dans l'obéissance du roi Edouard, son supérieur doit néanmoins le déplacer un temps vers un autre prieuré anglais. ${ }^{34}$ Chercher fortune au service de l'Eglise devenait hasardeux, les débuts de la guerre offraient des chances à qui avait le goût des armes, ce qui à n'en pas douter était le cas d'Elie.

Dès 1339 , il a franchi le pas. ${ }^{35}$ Bien vite, sa naissance et ses talents le font sortir du rang : ses premiers commandements militaires identifiables consistent à être capitaine de la garnison d'Auberoche en $1340^{36}$, puis châtelain de Bourg-sur-Gironde, dès 1341 probablement. Ce dernier poste n'a rien de prestigieux, mais comporte des responsabilités, et d'abord celle de surveiller la réparation des murs et des fossés de la place. En mars 1342, Elie n'a encore touché que le tiers des 1113 l. bordelaises dépensées à ces fins et le sénéchal Olivier de Ingham doit intervenir pour qu'on lui paie le reste. ${ }^{37}$

Elie fait bien vite connaissance avec les ennuis ordinaires des chefs militaires au service du roi-duc qui promet beaucoup mais paie peu: le mandement du sénéchal est inclus dans un billet d'Antonio Uso di Mare constatant que le 28 septembre 1343, presque la moitié des sommes engagées restaient dues. ${ }^{38}$ L'affaire traînait encore en 1365 , de même que beaucoup d'autres impayés! Son seigneur se soucie néanmoins de pourvoir à son état, le service militaire doit remédier à son défaut de fortune, dès le $1^{\text {er }}$ juin 1342, Elie reçoit son premier fief-rente : $200 \mathrm{l}$. tournois concédées sur la coutume dès $1342 .{ }^{39}$ A ce moment, commence un processus qui va durer plus de vingt ans : Edouard III ou son fils accumulent

languedociennes et provençales et pourraient se rattacher à la branche fidèle aux Valois, ou bien n'avoir aucun rapport même avec elle, sans parler de Guillaume, prieur de Cluny puis abbé de Montiéramey ( $\left.{ }^{\circ} 11078,25200,26619,27788\right)$

33 Calendars of Entries in the papal Registers relating to Great Britain and Ireland, t. II, éd.

W. H. Bliss, Londres 1895, pp. 326-328, 335, 348.

$34+$ Calendars of Fine Rolls, t. V, p. 248 (novembre 1341).

35 E368/138, Communia Recorda, Saint-Michel 40, mbr 15-21, pièce 16.

36 Froissart, Chroniques, t. II, p. 48;

37 Capra, Une carrière anglo-gasconne, p. 58 et n. 7.

38 E368/138, Communia Recorda, Saint-Michel 40, mbr 15-21, pièce 40.

39 Capra, Une carrière anglo-gasconne, p. 61. 
les promesses de versements et grâces sur la tête de leur cher et fidèle Elie, et les honorent fort peu. Ses frères Guillaume-Sanche comme Jean, et beaucoup d'hommes d'armes, réclament eux-aussi de façon chronique leurs gages de guerre en souffrance. ${ }^{40}$ On peut supposer que de son côté Elie a dû fortement s'endetter par moments.

La chose n'a rien que de très banal, Arnaud de Durfort, pères et fils, de fidèles vassaux d'Edouard III, vers 1325-1355, couraient aussi après leurs soldes en retard; les promesses de fiefs-rentes, supposés étaler dans le temps les obligations financières de leur seigneur, n'avaient guère plus d'effet. Il fallait emprunter, mais les Durfort trouvaient des bailleurs de fonds, et réussissaient de temps en temps à obtenir enfin leur dû en se rendant à Londres et en touchant de l'Echiquier ce que la connétablie ne pouvait leur verser. Un homme influent a du crédit, que l'on songe à Miles de Noyers, et d'autres grands seigneurs de la cour de Philippe VI! ${ }^{41}$. Nicole de Peña se demandait si dans la première moitié du XIVe siècle les vassaux gascons des zones frontalières avaient ou non «choisi le camp qui pouvait leur assurer les plus grands profits», et montrait que la réponse à cette question était ambiguë dans la mesure où la faiblesse des ressources de la connétablie et le désordre d'une Aquitaine administrée de trop loin entretenaient une pagaille qui profitaient aux plus entreprenants. ${ }^{42}$ On peut certainement étendre cette démonstration à propos des Durfort à Guillaume-Sanche et Elie de Pommiers, sans toutefois minimiser la très réelle et très puissante fidélité à leur seigneur naturel, en laquelle ils surent trouver leur profit. En tout cas, les écuyers nommés Pierre et Amenion de Pomes qui figurent avec de bien modestes montures dans la montre présentée par Jourdain de Lisle à Moissac en 1352 pour le roi de France, en compagnie d'autres écuyers gascons ou languedociens, n'ont pas fait fortune, non plus que que l'écuyer nommé Pierre de Pommiers qui figure en tête d'une retenue de 25 écuyers à Condom en $1369^{43}$.

\section{Deux styles de carrières militaires : celle d'un vassal et celle d'un professionnel de la guerre?}

Guillaume-Sanche a conclu six contrats militaires pour la dëfense du duché entre 1354 et $1360 .^{44}$ Il sait se faire écouter pour obtenir son dû car en 1357, Edouard III insiste auprès du connétable afin que celui-ci règle ses gages à Guillaume-Sanche. ${ }^{45}$ et il obtient les récompenses dues à sa fidélité. Il exerce à ce moment, mais pour la seule fois de sa vie, un commandement important. En 1354, le Soudan de La Trau reprit Coutras, Abzac et Pomerol, le sire de Pommiers reçut la garde de ces trois places, ce qui lui posa de sérieux problèmes de ravitaillement. ${ }^{46}$ On se demanderait même si l'action du Soudan n'a pas été un renfort décisif apporté à ces opérations militaires, entreprises auparavant par Guillaume-

$40 \mathrm{C} 61 / 69 \mathrm{mbr} 10, \mathrm{C} 61 / 70 \mathrm{mbr} 17$ etc.

41 R. Cazelles, La société politique et la crise de la royauté sous Philippe VI de Valois, Paris, 1958, pp. 395-397.

42 Peña, Vassaux gascons au service du roi d'Angleterre, pp. 5-21.

43 B. N. NAF $8603 n^{\circ} 18-22$ et 71, la retenue de 1369 comporte plusieurs bâtards et on n'y remarque surtout des noms gascons faisant prenser aux terres des Armagnac plutôt qu'au Bordelais.

${ }^{44}$ E101/168 $12 n^{\circ} 29,37,4459$ et $75 ; E 101 / 1693 n^{\circ} 17,19$ et $26 ; 650 n^{\circ} 31,63$ et 142. Il y a en outre dex reçus dont les ordres de paiement n'existent plus, E101 $650 n^{\circ} 79$ et 103.

$45 \mathrm{C} 61 / 69 \mathrm{mbr} 10(30 / 12 / 1357)$.

46 E101/169 2,I, n ${ }^{\circ} 4$ et 8 . E372/207 fol. $17 \mathrm{v} \mathrm{mbr} \mathrm{2,} \mathrm{fol.} 18 \mathrm{r} \mathrm{mbr} 2$. 
Sanche, en tout cas ce dernier avait conduit le siège de fortification ennemies près de Libourne. ${ }^{47}$

En 1352, Guillaume-Sanche avait réussi à contracter une union fort avantageuse avec Jeanne, héritière présomptive du vicomte Raymond IV de Fronsac ${ }^{48}$, Edouard III avait certainement favorisé ce mariage entre un fidèle sûr et la fille d'un vicomte regardant plutôt du côté des Valois. Par sa naissance et son alliance, Guillaume-Sanche mérite pleinement le titre de noble et poderos baron ${ }^{49}$. Déjà lointain parent des Grailly ${ }^{50}$, il cousinait désormais avec les Albret, la mère de son épouse étant une des filles d'Amanieu VII. ${ }^{51}$ A vrai dire, avant même ce moment, Guillaume-Sanche semble déjà assez liẻ avec certains membres de cette famille, puisqu'il a été un des exécuteurs testamentaires de Bérard $\mathrm{I}^{52}$, et qu'on le trouve en 1350 témoin d'une obligation du sire d'Albret. ${ }^{53}$

La femme de Guillaume-Sanche lui apporta aussi Belin et Uza en héritage, comme petite-fille de Yolande del Soler. Cela changea le sort de Guillaume-Sanche, le médiocre seigneur bazadais devenait riche homme, il mettait ainsi la main sur une nuée de possessions englobant jusqu'à l'échouage des baleines et l'exploitation des épaves dans la région de Biscarosse, Saint-Julien et Soort, d'où un litige avec le sire d'Albret : Bernard d'Albret ayant jadis reçu du roi-duc les droits ducaux à Mimizan et en Born, les redevances du domaine en question devenaient difficiles à percevoir. La querelle se limita à une procédure per legitimam informationem, en mars $1358^{54}$, simple enquête administrative du sénéchal ${ }^{55}$, fort idoine au cas de barons n'aimant guère que le sénéchal regarde de trop près leurs affaires de famille ; on ne saurait mieux dire que Guillaume-Sanche était désormais un homme de très haut rang. La forte situation de Guillaume-Sanche lui permettait de tenir tête au jeune sire d'Albret, après avoir fait de mème avec son père. En effet, quelques années auparavant, Derby avait confié le château de Castets-en-Dorthe à Bernard d'Albret, qui l'avait donné en garde au sire de Budos; quand ce dernier entra en rébellion; GuillaumeSanche, comme allié de Bernard avait repris la place, mais refusé de la rendre d'où une procédure per legitimam informationem le 14 juillet 1355.56

Pendant ce temps, Elie s'est s'engagé complètement au service du roi-duc; à la différence de son frère, il avait impérativement besoin de continuer à faire carrière. En 1347, Elie qui doit avoir au moins la trentaine, décroche un poste important: Thomas Cook le désigne comme sénéchal de Périgord le 13 avril 1347, Edouard III confirme cette

47 E372/199 fol. 39r mbr 2.

48 En mars 1354, Jeanne est considérée comme dame de Fronsac, C61/66 mbr 15.

49 Arch. hist. de la Gironde, t. XVI, p. 81, acte du 19 juillet 1353 (copie du XV ${ }^{\mathrm{e}}$ siècle).

50 Ruault, La famille de Pommiers, p. 87.

51 Soulé, Le duché de Fronsac, des premiers vicomtes aux ducs de Richelieu, Bordeaux, 1941.

52 Calendar of Patent Rolls, 1345-1348, p. 287, 289, 331, 427, 432, ce ne fut pas une sinécure en raison d'un procès fleuve contre le prieur de Bustleham et l'évêque d'Ely.

53 Arch. hist. de la Gironde, t. IV, p. 110.

54 C61/70 mbr 16, 24/05/1358.

$55 \mathrm{Au}$ contraire de la procédure audita querela qui est proprement judiciaire, Capra, $L$ 'administration anglo-gasconne, p. $146 \mathrm{n} 16$.

$56 \mathrm{C} 61 / 67 \mathrm{mbr} 4$; les querelles à propos de la juridiction de Castets-en-Dorthe n'étaient pas nouvelles, déjà en 1274 il en est fait mention, Ruault, La famille de Pommiers, p. 35. 
nomination le 4 août 1348.57 Cette jolie réussite personnelle et la peste qui fait des héritières inattendues expliquent sans doute l'établissement d'Elie: en 1349, nous trouvons pour la première fois notre homme appelé seigneur d'Arbanats, il avait épousé une petite nièce de feu Clément V. ${ }^{58}$ Elie ne fait pas un mariage aussi brillant que Guillaume-Sanche, mais inespéré néanmoins pour un cadet, il tient désormais un rang très honorable dans la noblesse du Bordelais. Et en partager aussi les soucis: il dut affranchir des questaux à Portets contre un fort versement en $1366 .{ }^{59}$

Elie devenu seigneur d'Arbanats, Amanieu a dû acquérir la seigneurie de Faleyras de la même façon ${ }^{60}$, on le trouve plus tard marié à Marie de Masdurand ${ }^{61}$, Jean devient à son tour seigneur de Cabanac vers $1362^{62}$ et Bertrand épousa Peyronne d'Argadens ${ }^{63}$, seul Arnaud resta sans alliance, du moins sans alliance connue. Cinq frères s'établirent, plus ou moins brillamment, et trois d'entre eux, Guillaume-Sanche, Elie et Bertrand laissèrent une postérité. C'est à ses cadets surtout que vont les dons de Guillaume-Sanche qui n'a manifestement pas boudé les succès de ses jeunes frères En tout cas, loin d'être ruinée par une pléthore de fils, la vigoureuse nichée des Pommiers a finalement profité de la terrible conjoncture démographique des années 1350-1360.

La dignité de sénéchal compensait le défaut de fortune d'Elie, dans la mesure où elle lui donnait l'espérance d'une belle carrière, mais dans l'immédiat elle ne lui valait que de faibles avantages matériels. En effet, son «fief» annuel de 100 livres sterlings, assigné d'abord sur les biens des bannis, ne lui est pas payé régulièrement; un reliquat de 1300 écus d'or, valant $1300 \mathrm{l}$. bordelaises, et trois chevaux, et constate par trois billets d'Antonio et Niccolo Uso di Mare, ainsi que onze lettres d'Olivier de Ingham, est payé en partie sur une fraction des $1000 \mathrm{l}$. versés par Stretley après 1354. Puis, comme décidément les ressources de l'incertaine sénéchaussée de Périgord ne suffisent pas et, de guerre lasse, on procède à une nouvelle assignation, sur les revenus de l'ensemble du duché cette fois, le $1^{\mathrm{er}}$ février $1358 .{ }^{64} \mathrm{Sa}$ sénéchaussée a apporté à Elie un titre, des occasions de profit, et du crédit, plus que des gages; elle a aussi fait de lui un chef de guerre dans un poste où ne manquait certes pas les responsabilités administratives et judiciaires, encore que cet aspect de son activité ne soit documenté que pour la fin de son dapiférat, vers 1358/1361, époque plus calme sur place que les années qui précédaient, et plus propices donc à l'épanouissement de missions «civiles» ${ }^{65}$, son rôle de sénéchal a été surtout guerrier.

57 Copie de la vidimation par Cook, E368/138, Communia Recorda, Saint-Michel 40, mbr 15-21, pièces 10, 24, 26 ; Une carrière anglo-gasconne, p. 58 et n. 8.

${ }^{58}$ E368/138, Communia Recorda, Saint-Michel 40, mbr 15-21, pièce 27 ; Une carrière anglo-gasconne, p. 58 et n. 9.

${ }^{59}$ En 1348, élie a aussi obtenu le droit de cosntruire une bastide à Portets, beau projet de nouveau seigneur qui n'eut aucune suite; Arch. hist. de la Gironde, LI, pp. 186-228.

60 Capra, L'administration anglo-gasconne, p. 672 n. 97 réf. absente, seigneur de Faleyras.

61 Second mariage? Ruault, La famille de Pommiers, p.157.

62 Léo Drouyn, La Guienne militaire, pp. XLV-XLVII.

63 Ruault, ibid., p. 157.

${ }^{64}$ Une carrière anglo-gasconne, pp. 59-60 et n. 20-23; E368/138, Communia Recorda, Saint-Michel 40 , mbr 15-21, pièces 13,14;C61/70 mbr 20. les promesses avaient commencé dès mars 1349 du fait d'Henry duc de Lancastre, lieutenant d'Edouard III, C61/72 mbr. 7 , confirmation du 20 mars 1351 .

65 Une carrière anglo-gasconne, p. 60 et n. 25-29. 
Devenu un homme établi, Elie gardait le goût des armes et on le voit beaucoup plus que son frère aîné se multiplier aux frontières du duché à la tête de fortes troupes. Dès le 4 janvier 1350, retenu par Chiverston, Elie conduit 19 hommes d'armes et 50 sergents à cheval $^{66}$; puis le 8 mai 1354 , une levée permanente de 29 des premiers et 100 sergents à pied. A plusieurs reprises, Elie reçoit en plus la direction d'autres retenues, temporaires en principe, 223 jours en 1350, 221 jours de juillet 1352 à mars 1353 pour un complément d'escorte de 29 hommes d'armes et 30 sergents, reconduit 277 jours jusque fin 1353 , et enfin 115 jours fin 1353/ avril 1354. La troupe reprend du service de mai à avril 1355. P. Capra notait «on se demande ce qui advint ensuite.» ${ }^{67}$ Mais la question dépasse peut-être le seul cas d'Elie de Pommiers. En tout cas, Elie de Pommiers a joui de la confiance de Chiverston comme de Stafford et conduit pendant trois ans une troupe d'une petite soixantaine d'hommes d'armes et de 150 sergents, ce qui est loin d'être négligeable. Pour autant, les ennuis d'argent d'Elie continuaient, à voir les rappels à payer ses gages.

Pendant ce temps, Elie continue ce que l'on pourrait appeler une «carrière militaire», où il récolte moins d'honneurs que son aîné, et court certainement plus de danger. La quarantaine bien sonnée, lui qui a souvent commandé des troupes d'une centaine de combattants, mais n'a jamais participé à des événements militaires de très grande envergure, se trouve désormais mêlé pour quelques années à des opérations majeures. Il fait partie de l'expédition d'Aiguillon en juin 1354, et ensuite se rend spécialement sur les frontières de sa sénéchaussée et à Castelmoron. Chiverston donna ordre de le payer le 4 juillet 1354, mais 11 ans plus tard cela restait encore à faire! En 1355, il participe à deux engagements. ${ }^{68}$

En juillet 1355, Chiverston le place d'urgence à la tête de la plus puissante forteresse de sa sénéchaussée, Bergerac, mais au bout de deux mois, le sire de Mussidan le remplace $^{69}$, à ce moment l'expédition du Prince Noir a déjà pris la mer, Elie doit vouloir s'y joindre; il est de la chevauchée de l'hiver et nous le retrouvons à Poitiers. Cet homme d'armes expérimenté a dû être un subordonné utile, un conseiller et un agent d'exécution avisé, pour un prince qui voyait grand mais était bien jeune.

\section{Guillaume Sanche et son cadet à l'âge mûr}

Gloire et profits de guerre

Lors du siège d'Aiguillon, en juin 1354 Guillaume-Sanche et Elie conduisaient l'un 65 hommes, l'autre 53, plus du quart des effectifs engagés, Guillaume-Sanche menait la plus grosse retenue, et les troupes de chacun surclassaient la modeste moyenne de 15/16 hommes conduits par les 25 capitaines; les deux frères ont rang et gages de barons, et sont

${ }^{66}$ E101/171/4/I n 25 ; E368/138, Communia Recorda, Saint-Michel 40, mbr 15-21, pièce 25 ,

67 Capra,Une carrière anglo-gasconne, p. 60 et n. 24.

68 Capra, ibid., p. 59 et n. 13-17.

${ }^{69} \mathrm{E} 101 / 168 / 12 \mathrm{n}^{\circ} 8,11$. 
les seuls à compter quelques chevaliers sous leur bannière. ${ }^{70}$ Le lignage des Pommiers avait pratiquement deux têtes, le talent militaire d'Elie avait fait de lui un baron, et s'il n'est pas l'égal de son frère, il est un homme de grand poids, tandis que Bertrand et Jean poursuivaient des destins plus obscurs.

Leur participation aux équipées du Prince Noir conforte cette hiérarchie entre les frères. Hewitt faisait de Jean et Amanieu de Pommiers de possibles acteurs des chevauchées du Prince Noir, rémunérés comme tels par des rentes et pensions; P. Capra, sur la base de particulars et du compte enregistré du connétable John Stretley a jeté le doute sur la seconde affirmation. ${ }^{71}$ Amanieu ${ }^{72}$, Bertrand ${ }^{73}$ et Jean ${ }^{74}$ semblent ne se signaler par aucune autre action d'éclat particulière, à ce moment du moins, que d'être de temps à autre les chefs de modestes retenues vers 1354/1358, et force est de constater que nous ignorons quand ils ont été introduits aux armes, probablement peut-être des années auparavant, obscurs écuyers combattant sous les ordres de Guillaume-Sanche ou Elie, ou de quelque autre chef.

Pourtant, si Jean et Amanieu ne font pas partie des dizaines de combattants, chevaliers ou écuyers qui ont bénéficié des prodigalités d'Edouard de Woodstock pour leurs bons services et particulièrement pour leur comportement à la bataille de Poitiers, ils ont certainement combattu sous ses ordres ou l'ont approché à un titre ou à un autre, car Amanieu semble avoir suivi le Prince en Angleterre en 1357 - dans l'été il lui vend des bijoux - d'autre part Jean a bénéficié d'un substantiel don de 63 l. sterlings en 1361, toujours en Angleterre ${ }^{75}$, et Froissart cite bel et bien Amanieu pour la chevauchée en Languedoc en 1355 et un raid en Berry en $1356 .^{76}$

Une fois de plus, Guillaume-Sanche et Elie occupent le devant de la scène au moment de la campagne de 1356: Richard d'Avesbury signale que le prince a sollicité Guillaume-Sanche d'y participer ${ }^{77}$, Froissart montre le sire de Pommiers dans la bataille qui affronte celle de Jean II, avec le Soudan de La Trau, le sire de Mussidan etc, et ensuite Guillaume-Sanche se fait payer très cher pour laisser partir ses prisonniers, il serait ensuite un des quatre barons laissés sur place par le prince pour gouverner le pays ${ }^{78}$, ce qui n'est peut-être pas tout à fait exagéré, car curieusement Guillaume-Sanche ne fait pas partie des seigneurs gascons qui ont afflué en Angleterre dans les mois suivants, pour aller faire la roue auprès de leur seigneur et traiter leurs affaires de rançon. ${ }^{79}$ Froissart aime à s'attarder

${ }^{70}$ Capra, Le siège d'Aiguillon en juin 1354, Villeneuve-s/Lot et l'Agenais. Féd. hist du SudOuest. Féd. des Soc. acad. et sav. Languedoc-Pyrénées-Gascogne, Agen, 1962, pp. 204-206.

${ }^{71}$ M. Hewitt, The Black Prince's expedition of 1355-1357, Manchester, 1958, et Capra, Les bases sociales du pouvoir anglo-gascon, p. 291 et n. 123.

72 Capra Les bases sociales du pouvoir anglo-gascon, p. 279 n. 31, 1 contrat E101/650 n 99.

73 Un contrat E101/169, II, $n^{\circ} 98$.

${ }^{74} \mathrm{E} 101 / 168,12 \mathrm{n}^{\circ} 76,85$.

75 Register of Edward the Black Prince, t. IV, Londres 1933, pp. 245-246, 251-252, 323.

76 Chroniques, éd. S. Luce, G. Raynaud, A.Mirot, S.H.F., Paris, 1869-1967, IV, p. 160, 175.

77 De gestis mirabilibus Regis Edwardi tertii, Londres 1889, p. 449.

78 Froissart, Chroniques, V, p. 32, 80.

${ }^{79}$ Cf Register of Edward the Black Prince et Issue rolls de 1357/1358, E403, 389, 390, 391, on repère Raymond de Pellegrue, Bertrand de Budos, le sire de Montferrand, Amanieu de Montpesat, Elie de Pommiers; d'autre part en mars 1358, Arnaud de Durfort, Amanieu du Foussat, Amanieu de Montpezat, le sire de Caupenne et Pons d'Andiran assistent au conseil du roi en Angleterre, E403/390 fol. 37. 
sur les chefs de lignage, c'est pourtant encore un fois Elie qui est un des héros heureux aux armes plus que son aîné, car il a fait une prise superbe : un des personnages rachetés à prix d'or par le prince pour Edouard III.

Elie connaît ainsi des disponibilités financières bien inhabituelles, car il est partie prenante dans la rançon du comte d'Eu, descendant de Louis VIII et donc lointain cousin de Jean II, pris à Poitiers par lui-même et achetẻ par le Prince 30000 florins, le 21 mars 1357, somme péniblement honorée en 12 ans, après un premier versement très tôt en Aquitaine. Une fois de plus, Elie ne se décourage pas, mais doit désigner trois séries successives d'attournés pour toucher son argent à Londres: Montersin de Bä̈oran et Gautier de Rouffignac - licencié en droit qui est juge-mage de Périgord -, Pierre Ramafort seul, enfin Pierre Larigaudie et Guillaume Feral - les uns et les autres lui ont probablement avancé l'argent. ${ }^{80}$ Toujours est-il qu'Elie touche presque la moitié des 30000 écus avant juillet $1359^{81}$, manifestement très favorisé en cela par rapport aux autres combattants obligés de se désaisir de leurs plus belles proies. Il convient de souligner l'énormité des sommes en jeu. Un banneret était payé $4 \mathrm{~s}$ sterlings par jour, les années qui auraient comptẻ par extraordinaire 365 jours de solde, il aurait perçu 731 , et voilà qu'il était question de plus de 5000 1.! Une embellie générale permet à Guillaume-Sanche et à d'autres, par exemple Franck de Halle, de toucher des arriérés de soldes imposants. Elie ne se retire pas sur ce succès inespéré, car en en 1359 , il est encore sur la brêche. ${ }^{82}$

Depuis des années, il avait l'habitude de réclamer ses gages de guerre ou de fiefrente, tout ou partie impayés ${ }^{83}$, et de quémander pour se faire rembourser des chevaux ${ }^{84}$; il s'agit là d'une attitude caractéristique de ces années où les impayés de soldes atteignent des proportions inquiétantes, particulièrement pour les Gascons ${ }^{85}$. Il manifeste la même pugnacité pour défendre son modeste patrimoine personnel, en l'occurrence une partie de Siorac, en co-seigneurie avec Thibaud de Gensac et Pierre de Pommiers. Ces deux personnages, envoyés par le père d'Elie pour garder ce lieu si fâcheusement exposé - et peut-être pour cela même assigné à un cadet! - l'avaient au contraire livré aux Français ; Elie dut le relever à grands frais après sa reconquête, en 1359 , il se fait attribuer par le sénéchal John de Chiverston les parts des coupables ${ }^{86}$. Elie est partout, dans les rôles gascons comme dans les documents comptables, cet homme d'action et ce fidèle, réclame son dû avec énergie, dévoué, pugnace et âpre au gain. Aurait-il pu au reste agir autrement?

\section{Des barons fidèles : un vassal et un serviteur}

Au sommet de leur carrière, les aînés des frères Pommiers ne sont pas interchangeables, pour des raisons de talents comme de rang. Lorsqu'il s'agit de représenter

80 Capra, Une carrière anglo-gasconne, p. 59 et n. 18-19.

81 E403/390 mbr 19, 29, 33 ; E403/391 mbr 5; E403/392 mbr 15, 18 ; E403/395 mbr 39 ; $403 / 397 \mathrm{mbr} 7,16$.

$82 \mathrm{E} 101 / 650 \mathrm{n}^{\circ} 118$.

83 E372/199 M.39r mbr 2 versement de 1046 1. bord. par Charuels en 1352-1354.

84 E368/138, Communia Recorda, Saint-Michel 40, mbr 15-21, pièces 16 et 31(1339 et 1345).

$85+\mathrm{K}$. Fowler, «Les finances et la discipline dans les armées anglaises en France», Cahiers Vernonnais, t. 4, 1965, p. 82. ; Peña, Vassaux gascons au service du roi d'Angleterre, pp. 521 F. Legrand, Approche des armées anglo-aquitaines sous les derniers Plantegenêts, DEA, Bordeaux III, 1995, p. 259.

86 Capra, Une carrière anglo-gasconne, p. 58 n. 10. 
le roi-duc pour veiller à l'application sur le terrain des décisions diplomatiques, on a constamment recours à lui Guillaume-Sanche, baron de premier plan désormais, et à son talentueux cadet. $^{87}$ L'article sur «Les bases sociales du pouvoir anglo-gascon» donne quelques exemples de ces missions qui ne sont confiées qu'à pratiquement des personnages tout à la fois fidèles et de haut rang: surveiller l'application des trèves renouvelées en avril 1354, en Bordelais avec Bérard d'Albret et le sire de Montferrand ${ }^{88}$, etc. Quelques mois plus tard, nous retrouvons Guillaume-Sanche avec cette fois le captal de Buch et Elie pour réparer les attentats contre les trèves. ${ }^{89}$ En 1360, Guillaume-Sanche accompagne le sénéchal en Poitou et Saintonge pour une tâche analogue ${ }^{90}$, tandis qu'Elie a la responsabilité de s'occuper des forteresses de Périgord, Quercy et Agenais ${ }^{91}$, puis suit John Chandos en Périgord, ses premières responsabilités de ce type remontant à 1358 en Berry. ${ }^{92}$ Enfin leur frère Amanieu de Pommiers reçoit mission de s'occuper des attentats contre les trêves en 1361.93

Pour des tâches sans particulière connotation militaire, Guillaume-Sanche passe indiscutablement avant Elie; il a l'insigne honneur de siéger dans un appel de France: il figure par trois fois dans les quatorze commissions qui entre 1354 et 1358 ont connu des causes portées en appel devant Edouard III comme roi de France, avec Plassan, avec le maire de Bordeaux, le conseiller Jean de Saucats, alors que son frère Elie intervient une seule fois de même que Bertrand de Montferrand et Jean de Grailly ${ }^{94}$; en 1358 avec Arnaud-Garcie du Foussat, sénéchal d'Agenais, Géraud du Puch, évêque de Bazas, Jean de Grailly et Bertrand de Montferrand, il est chargé de connaître le cas particulièrement grave du conseiller Pierre de Rouffiac, emprisonné par John de Chiverston. ${ }^{95}$ Il est des quelques grands seigneurs du duché auxquels le pape s'adresse, en 1357, pour faire libérer les chevaliers du vicomte de Turenne capturés pendant une trêve par Bertucat d'Albret, au cours d'un voyage auprès de la cour pontificale. ${ }^{96}$ Enfin, au début du pontificat d'Urbain $V$, Hélie, Amanieu et Jean, tous chevaliers, figurent dans le club très fermé des seigneurs et clercs de haute volée qui reçoivent le privilège d'avoir un autel portatif et de faire dire un office privé avant le lever du jour, on trouve là le seigneur de Cabanac et celui d'Arbanats en une compagnie très relevée; leur parent Raymond, aussi chevalier, et sa femme Assahilde recevaient au même moment un privilège de nature à les classer parmi les gens de qualité : recevoir l'absolution in articulo mortis d'un prêtre de leur choix..$^{97}$

Occasionnellement, Guillaume-Sanche a eu des tâches «diplomatiques», mineures à vrai dire ; en son jeune temps en 1343-1344, il accompagne en Castille, Jean de Brocas, un des responsables des écuries royales, pour ramener des genêts d'Espagne à son seigneur et

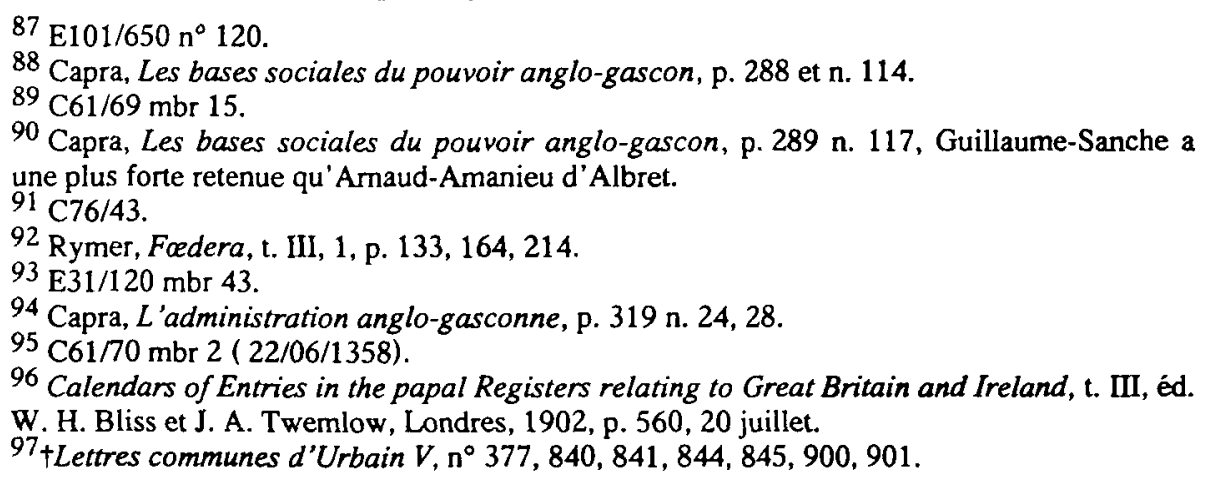


roi $^{98}$; il est bien possible qu'en la circonstance sa qualité de grand seigneur ait été doublêe de quelque compétence technique, car les sires de Pommiers de même que de Durfort, Lesparre et Bergerac, devaient avoir des êlevages de chevaux réputés, à voir les comptes de l'Equitium Regis, une génération plus tôt il est vrai. ${ }^{99}$. Il est des négociateurs et otages que le Frince Noir envoie en 1364 auprès de Gaston Fébus pour obtenir la libération du sire d'Albret, son parent par alliance. 100

La cérémonie mise sur pied à la cathédrale Saint-André en juillet 1363 pour entendre le premiers serments prêtés au nouveau prince d'Aquitaine constitue un gros effort de propagande; la mise en scène fait paraître 19 barons, 19 chevaliers et 19 écuyers, Guillaume-Sanche s'avance le premier après Arnaud-Amanieu d'Albret pour prêter hommage au Prince Noir, parmi ceux que l'on qualifie de barons. ${ }^{101}$ Le seigneur de Roquetaillade s'intercale entre lui et Elie, Bertrand suit ses aînés à quelque distance, $9^{\mathrm{e}}$ des barons. La famille de Pommiers pouvait avoir quelque sujet de fierté avec trois barons. Jean de Grailly aurait pu être le second, mais il n'est pas là. Le sire de Montferrand ne passe que le $16^{e}$ des barons, mais il a tout récemment accédé au titre et ne peut encore prétendre aux égards dont son père jouissait de par sa position personnelle. Dans les dix-huit barons qui suivent Arnaud-Amanieu, les cinq premiers puis les $8^{\mathrm{e}}, 15^{\mathrm{e}}$ et $16^{\mathrm{e}}$ sont des fidèles actifs, le $6^{\mathrm{e}}$ un mineur en tutelle. Gaillard de Durfort, rallié récent est mis en $17^{\mathrm{e}}$. Auger de la Motte $\left(7^{\mathrm{e}}\right)$, Raymond de Fargues $\left(9^{\mathrm{e}}\right)$, Raymond-Guillaume seigneur de Castets $\left(10^{\mathrm{e}}\right)$, Bertrand de Cases $\left(11^{\mathrm{e}}\right)$ Pierre de Gabarret $\left(12^{\mathrm{e}}\right)$, Thibaud seigneur de Budos $\left(4^{\mathrm{e}}\right)$ et Jean de Lalande seigneur de La Brède (18 $)$ n'ont jamais paru à l'occasion des contrats de guerre. Il peut y avoir là, tout en marquant la supériorité des meilleurs partisans, un souci de dosage des honneurs. En tout cas, on voit que des nobles de très bon rang ne participent pas personnellement aux guerres, ni même aux chevauchées, en tout cas pas comme chefs de retenue. La place éminente de Guillaume-Sanche tient à ses titres, à sa fortune, mais aussi à ses bons services.

Alors qu'Elie devait toujours supplier son seigneur qu'il veuille bien le payer, Guillaume Sanche vivait du sien, et sans doute assez bien. Il ne voit pas d'inconvénient à consentir des dons de valeur à son frère Jean. Il confirme l'attribution faite par son beaupère à Ayraud Albert de tous les droits possédés à La Rivière ${ }^{102}$ Guillaume-Sanche a les moyens d'être libéral.

Jusque vers 1354 , Guillaume-Sanche a reçu quelques dons de son prince ; ils pèsent lourd, cependant notre homme ne paraît jamais avoir étroitement dépendu du pouvoir par des avantages financiers très poussés, il ne touche ni rente ni pension sur la connétablie ou l'échiquier. Les grandes familles, du moins ceux de leurs membres qui avaient une assiette seigneuriale suffisante, s'engageaient activement au service de leur prince pour d'autres raisons probablement que de simples cadeaux du prince : elles y gagnaient évidemment en influence, sinon en indépendance. Son frère Elie a beaucoup plus activement servi son prince, et beaucoup plus reçu de lui, mais au prix sans doute d'une subordination notoire.

\footnotetext{
98 Calendar of Close Rolls, 1343-1346.

${ }^{99}$ E101/101/6 ; E101/101/8; E101/101/16 (1315-1335 environ) ; cf A. Ayton, Knights and

Warhorses. Military Service and the English Aristocracy under Edward III, Londres, 1994.

100 Arch. hist. de la Gironde, t. IV, p. 127.

101 J.-P. Trabut-Cussac, Le Livre des Hommages d'Aquitaine, Bordeaux, 1959, pp. 73-74 ;

Capra, Les bases sociales, pp. 297 -298 n. 142.

102 C61/70 (ou 66 plutôt) mbr 15, 30/03/1354.
} 
En dehors des contrats militaires et sauf le don de la coutume de Royan, GuillaumeSanche ne fait que de modestes apparitions dans les écritures des caisses londoniennes et bordelaises, mais pour des mouvements de fonds importants, puisqu'il reçoit $331.6 \mathrm{~s} .8 \mathrm{~d}$. sur le remboursement d'une avance, puis une assignation de $200 \mathrm{l}$. sur les coutumes de Londres. Tout cela se passe au moment où se règlent les grands succès gascons ${ }^{103}$, notre homme avait des liquidités, venues peut-être de rançons. Ses ressources qu'il sait opportunément mettre à disposition de son prince peuvent lui venir de participation à des affaires commerciales. En tout cas à ce moment, il ne touche par ailleurs que quelques gages de guerre avec son frère Elie. ${ }^{104}$

\section{Retraite ou fausse sortie vers $1360 / 1361$ ?}

Sur la fin de sa longue carrière Elie bénéficie de lettres de protection royale ${ }^{105}$; et en 1365, il finit par obtenir le règlement de ses créances sur le roi-duc, vieilles de parfois vingt-trois ans. Un long document qui renferme quarante-deux pièces répertorie toutes les obligations qui font l'objet de cette transaction pour solde de tout compte ; elle porte entre autres sur les $200 \mathrm{l}$. tournois de rente assignées sur la coutume de Bordeaux en 1342, sur une autre rente de 100 marcs allouée par Derby en 1349 sur des revenus fiscaux en Périgord et enfin, sur le plus récent et le plus subtantiels des dons princiers, soit une rente de 2001 . sterlings, dont la moitié héréditaire, en 1358.106 Les caisses de Bordeaux ou de Westminster avaient fini par honorer les gages de guerre et autres impayés afférant à des fonctions remplies par Elie, mais non ce que le prince lui avait promis à titre de récompense, et s'il entendait se retirer et ne pouvait plus prétendre à des gages, il importait qu'on lui servît effectivement le fruit des grâces royales.

Le 10 octobre 1365, après de laborieuses tractations en cours depuis juillet avec la trésorerie ${ }^{107}$, Elie touche 1000 marcs soit une petite partie des sommes dues, mais il s'agissait de créances désespérées, et il fallait être un bon serviteur bien en cour pour tirer quelque vaillant d'Edouard III. Enfin, le 14 octobre 1365, Elie reçoit un fief - rente héréditaire - privilège assez rare — sur les revenus de la principauté ; l'enregistrement de l'acte reproduit un préambule inhabituellement ronflant où il est question de «l'ornement de la noblesse et de la parfaite constance dans la loyauté qui se sont trouvés sans faute dans la personne de notre cher et fidèle Elie»; ${ }^{108}$ Puis, nouvelle marque d'estime, il reçoit une coupe et une aiguière d'argent ${ }^{109}$, prise de congé princière : le Prince Noir ne laissait pas repartir au pays les chefs les plus appréciés de ses mercenaires allemands, sans leur donner en cadeau et en souvenir une jolie somme pour leur retour et une belle coupe d'argent pleine de nobles d'or. ${ }^{110}$ A supposer que le nouveau fief-rente d'Elie soit payé, cela signifiait aussi que le gouvernement ducal aiderait la famille d'Elie à maintenir un rang et

\footnotetext{
103 E401/443 mbr $20(9 / 02 / 1358)$ et E401/449 mbr 23 (14/03/1358) reçus avec sceaux pour une autre affaire E213/247 et 260 .

104 E403/392 mbr 8 8/05/1358.

$105 \mathrm{C} 61 / 70 \mathrm{mbr} 20(1 / 02 / 1358)$.

106 Capra, Une carrière anglo-gasconne, p. 61 et n. 31-34.

107 Ibid., p. 62 n. 38.

$108 \mathrm{C} 61 / 78 \mathrm{mbr} 2$.

109 Ibid., p. 61 et n. $35-37$.

${ }^{110}$ Register of Edward the Black Prince, t. IV, pp. 250-252.
} 
un train de vie fort supérieur à ses revenus patrimoniaux. La reconnaissance pour un vassal doit s'inscrire dans la durée et faciliter le renouveau de bons services aux générations futures.

Pour ce qui apparaît bien comme une cessation d'activité au bout de trente ans de bons services, vers cinquante-cinq ans au minimum, Elie récupérait moins du quart de ce qu'on lui devait et s'enrichissait d'une ultime promesse censée arrondir l'héritage qu'il transmettrait, et d'un beau souvenir princier. Mais il restait en compte avec son seigneur pour la rançon du comte d'Eu.

Guillaume-Sanche et Elie reviennent sur les champs de bataille en 1367 pour y jeter leurs derniers feux en Castille, sous les ordres de leur prince. Elie est d'abord envoyé consulter Edouard III en Angleterre, mais on le retrouve bientôt à Najera avec son aîné, qui à cette occasion a peut-être fait un prisonnier, mais il faut reconnaître qu'à ce moment d'autres ont ravi la vedette à Guillaume-Sanche et Elie. ${ }^{111}$ Ensuite, ce dernier a peut-être pris part à un siège de Montpont en $1371^{112}$, puis il n'y a plus trace de lui.

A ce moment, la famille de Pommiers n'occupe plus le devant de la scène, Guillaume-Sanche et Elie sont définitivement retirés ou déjà morts, leurs enfants probablement trop jeunes; Bertrand et Amaud occupent des postes modestes, l'un comme capitaine de Saint-Macaire, l'autre de La Réole, et de toutes façons, les finances du duché, complètement exsangues n'offrent pas de meilleure possibilité. ${ }^{113}$

\section{Une contribution à l'histoire de la noblesse gasconne}

Les frères Pommiers ont mené des carrières parallèles, mais distinctes, on devine trois fortes personnalités et non une meutes de cadets chassant dans le sillage de l'aîné. Leur cas sur le plan psychologique tendrait à prouver que le statut de puinés dans les familles nobles où pourtant la tradition du droit d'aînesse était fort vigoureuse, ne condamnait pas à une situation subordonnée, et n'ôtait ni le goût de l'action ni le sens de la réussite. Cette redoutable fratrie de chevaliers n'a pas connu semble-t-il de conflits graves, l'autonomie de chacun a créé les bases d'une saine entente.

Qu'on nous permette de prolonger le champ chronologique de P. Capra, pour mieux dégager la portée de ses travaux. A partir de 1363, Amanieu qui est certainement nettement plus jeune que Guillaume-Sanche et Elie et n'a pas aussi bien réussi socialement, a quitté le service d'Edouard III pour celui du roi de France, bientôt nous le trouvons sous la bannière de du Guesclin à Cocherel, avec d'autres Gascons du reste. Cet homme de guerre avait besoin encore d'un employeur quand ses aînés étaient déjà repus ou las, et il l'a trouvé par moments dans l'autre camp, ou en Italie au service du pape, somme toute là où il y avait des contrats de guerre intéressants. Auparavant, il avait suivi les voies de ses aînés. Au

111 Froissart, Chroniques, t. VI, p. 205, VII, p. 9, 49, Chronique de Sire Bertrand du Guesclin, éd. J. A. C. Buchon, dan Choix de chroniques et mémoires sur l'histoire de France, Paris, 1838, pp. 46-52 (chap. 79-87).

112 Froissart, Chroniques, t. VIII, p. 14.

113 Arch. hist. de la Gironde, t. XIII, pp. 331-335. 
cours des expéditions de $1355,1356,1359-60$, il est du combat, s'y distingue même; en 1361-1362, il commence à s'occuper de maintien de trêves. Sa route semblait toute tracée, mais la paix lui rogna les ailes. Il ne resta pas sur place où immanquablement il eût été sollicité par les aventures castillanes du Prince Noir après quelques années d'inactivité! Une péripétie antérieure, montrait qu'il s'agissait d'un personnage un peu moins commode à manier que ses frères, qui n'ont jamais posé de problèmes à l'autorité princière: il avait participé à l'assassinat d'Amanieu de Madaillan, avec Auger de Montaut, le Soudan de La Trau et quelques autres, c'est-à-dire en honorable, mais redoutable compagnie. ${ }^{114}$

De la solidarité des Pommiers nous ne savons pas grand'chose, si ce n'est l'influence qu'exerçait Amanieu, le dernier survivant ou l'un des derniers, sur le jeune Guillaume-Sanche IV vers $1376 / 1377$, et qui a très logiquement poussé son neveu Guillaume-Sanche IV à trahir. Amanieu jette une note discordante, parmi tant de chevaliers fidèles. On ne saurait voir ce groupe familial de façon monolithique, il est la somme de toutes les complexités de la noblesse gasconne, au gré des retournements de tendance militaire entre 1335 et 1375 .

Les aînés des frères Pommiers cessent leur activité militaire vers 1360, et ne refont surface que très brièvement en 1367 , voire 1370 ; c'est tout une génération qui se retire, après grosso modo le traité de Brétigny, seul Bertrand de Montferrand a vraiment fait florès dans le sillage du Prince Noir ${ }^{115}$, et encore assez peu de temps. ${ }^{116}$ Les compagnons d'armes qui savent se tailler la part du lion auprès du Prince, et obtenir de lui que, par un véritable coup de force, il impose au receveur de son père de leur verser de grosses sommes pour des arrérages de rente, s'appelaient Eymeric de Tastes, Raymond de Pellegrue, Petiton de Curton, Bertrand de Francs, chevaliers et Naudon de Rovignan , écuyer. ${ }^{117}$ Le seul qui ne date pas sa quittance de Lormont le $1^{\circ}$ août ou le jour suivant est Raymond de Pellegrue, il s'agit du seul aussi qui a assurément derrière lui une longue et belle carrière aux armes, il ne se joint pas aux proches du Prince. Un autre génération d'hommes d'armes faisait connaissance avec la valeur des promesses princières. Un des rares chefs de guerre gascons à avoir une carrière - presque exclusivement militaire— qui va des armées du Prince Noir et avant au règne de Richard II est Raymond Bernard —ou Bermond- de Preissac, Soudan de La Trau qui eut l'honneur rarissime pour un Gascon de devenir chevalier de la Jarretière en $1379^{118}$; il est vrai qu'on ne trouve guère trace de lui avant 1354 ni après $1381^{119}$ à la tête d'une troupe, ses vingt-sept ans de glorieux services dépassent un peu les vingt et quelques années continues relevées pour Elie de Pommiers. Il passé sans trop vaciller dans ses engagements politiques le cap des années les plus glorieuses et lucratives d'Edouard III et du Prince Noir, quand Amanieu allait chercher fortune ailleurs. Ces carrières d'hommes de guerre, suivies par le menu, doivent amener à écarter toute idée simple quant à l'attitude politique de la noblesse gasconne, voire à sa trop proverbiale versatilité, les choix ne sont sont pas posés dans les mêmes termes pour les hommes nés vers 1320 et pour leurs fils ou

\footnotetext{
114 Ruault, ibid., pp. 116-125. Rémission en 1355, Capra, L'administration anglo-gasconne, p. 545 n. $53,808-809$, n. 54-55.

115 Register of Edward the Black Prince, t.IV, p. 245 (dons d'armes).

116 Décès signalé dans les comptes du receveur de Saintonge1361/1362, E101/176/2.

$117 \mathrm{E} 101 / 176 / 20 \mathrm{n}^{\circ} 25-31$.

118 G. F. Peltz, Memories of the Order of the Garter..., Londres, 1841, p. CLIII, $\mathrm{n}^{\circ} 66$.

119 E101/68/197-199 et E101/70 2/614-615.
} 
leurs jeunes frères. On voit peut-être mieux aujourd'hui ce qui avait changé entre la génération de Guillaume-Sanche et Elie et celle d'Amanieu.

\section{Conclusions 1975-1995}

Chacun à sa façon, tous deux bien nés, Elie et Guillaume-Sanche sont des barons et ont une influence sociale majeure, l'un presque uniquement par le service du prince, l'autre en le conjuguant aux chances de son héritage et de son alliance. L'engagement politique de Guillaume-Sanche, fort de ses possessions seigneuriales, n'est pas moindre que celui de son talentueux mais impécunieux cadet, il lui avance d'ailleurs de l'argent, une seule fois, il est vrai, mais la chose était risquée! Leur réussite et finalement leur ascension sociale ne pouvait qu'arranger le prince qu'ils servaient, et ce n'était pas la première fois que ce phénomène s'observait, que l'on songe au cas, certes plus spectaculaire, des Grailly. Les rois-ducs n'ont jamais eu l'occasion ou l'intention de procéder à des fournées de confiscations pour caser des hommes sûrs dans leur duché, mais ils faisaient plus ou moins bien la fortune de leurs nobles les plus dévoués.

\section{Héritage et service}

Le plus grand nombre des capitaines dont les rétributions de guerre nous ont fourni les noms ne touchaient qu'assez rarement cadeaux et pensions, à l'image de GuillaumeSanche et au contraire d'Elie. P. Capra se demandait si ceux qui s'engagaient constamment avec le pouvoir et sollicitaient des secours, des récompenses, n'étaient pas gênés dans leurs relations avec les seigneurs ne recevant que des miettes, mais il faisait observer aussi que les nobles avaient, peut-être, l'esprit fort délié à cet égard. S'abandonner à la protection de son seigneur, s'en remettre à lui pour conserver ses droits ou recevoir des biens, était une première chose. Tomber en son pouvoir par des largesses acceptées, en était une seconde. Le clergé non plus ne recourait presque jamais au roi-duc mais pour des raisons bien différentes. Avec une vingtaine d'années de recul, nous nous permettons de reformuler les intuitions très justes de $\mathrm{P}$. Capra, quant aux ressorts de la fidélité et à la complexité d'attitude des générations de nobles qui ont vécu la "genèse de l'Etat moderne» dans le contexte féodal.

Guillaume-Sanche a été un vassal de son prince, son cadet plutôt un serviteur, mais comme vassal, le premier a été mis en un rang très supérieur à celui de sa naissance, de par son engagement persistant au service de son prince, et le second est devenu un vassal en vue. Ni l'un ni l'autre ne font les fréquents séjours londoniens qui permettaient aux Durfort de récupérer leurs créances, ou qui ont conduit leurs cadets et quelques jeunes loups gascons en Angleterte auprès du Prince Noir en 1357. Ils ont de la chevance, pensent peutêtre que «service a court n'est point vray heritage» 120 . Les recherches sur les noblesses françaises ou ibériques ${ }^{121}$ améneraient par contre à ne prêter aux nobles, même les plus grands, aucune répugnance à demander des dons lorsqu'ils savent devoir en obtenir, les

120 Proverbe cité par P. Contamine, L'Etat et les aristocraties, XII $-X V I I^{e}$ siècle, France, Angleterre, Ecosse, Paris, 1989, p. 21 et n. 30.

$121_{\dagger}$ Voir M. -Cl. Gerbet, les noblesses espagnoles au Moyen Age, XIe-XVe siècle, Paris, 1994. 
nobles gascons ont peu à espérer la plupart, sinon bouderaient-ils le pactole ? De toutes façons, la seule perception de gages de guerre représentait déjà un complément de ressources appréciable.

Or les Pommiers ont conduit parmi les retenues les plus notables. Les paiements militaires de 1354 montrent qu'Elie ${ }^{122}$ et Guillaume Sanche ${ }^{123}$ de Pommiers font partie de cette poignée de chevaliers qui entraînent plus de 25 hommes au combat avec Bertrand de Montferrand ${ }^{124}$, Arnaud d'Albret ${ }^{125}$ et les deux frères Gaston et Pierre de Gontaud. ${ }^{126}$ Il faudrait ajouter le sire de Caumont à cette liste, ainsi que Bérard II d'Albret. ${ }^{127}$ Pour le nombre de contrats militaires, seuls Raymond de Pellegrue et Agassac surclassent Elie, et de peu ${ }^{128}$. Et surtout, Elie réunit autour de lui des troupes importantes et d'un niveau social soutenu. Au cours de 1355, il mène 100 combattants, dont 5 chevaliers, 35 hommes d'armes (écuyers) et 60 sergents à cheval, en Saintonge puis en Agenais, soit le plus fort contingent levé cette année. Dans d'autres cas, on le voit flanqué de 65, 54, 30, 24 et enfin 15 hommes.

Les chefs militaires les plus souvent retenus n'appartiennent pas au tout premier rang de la noblesse gasconne, Elie le cadet a plus combattu que son aîné plus fortuné, on ne peut donc qu'adhérer à cet égard aux appréciations de $\mathrm{P}$. Capra sur la réserve des plus grands barons, mais en songeant aussi que le sénéchal préférait certainement des chefs militaires plus dociles que de trop grands personnages, et que le goût des armes et le talent militaire sont affaires de personnes. La noblesse gasconne vers $1340-1360$ a toujours une identité profondément guerrière, mais déjà l'exercice d'un véritable métier des armes interfère avec la hiérarchie de naissance.

Il convient toutefois de regarder au-delà des chefs et de penser à la tourbe anonyme des hommes d'armes qui les suivent. Au contraire de P. Capra, nous trouvons la noblesse gasconne fort guerrière; bien sûr, beaucoup des vassaux qui prêtent hommage en 1363 n'ont pas été chefs de retenue, mais leurs fils, leurs frères ou eux-mêmes doivent faire nombre dans les retenues des Gontaud, Pommiers, Pellegrue et autres. Avec Elie et ses puinés, on vérifie l'enthousiasme militaire des nobles, remarqué par P. Contamine et aussi

122 Paiement du 18/06/1354: 19 hommes d'armes dont 3 chevaliers et 37 sergents à pied E101/650 $n^{\circ} 534 / 07 / 1354$; Senebrun de Curton 20 hommes armes et 30 sergents 18/06/1354 E101/169/1 n $n^{\circ} 14$.

123 Paiement du 20/04/1354: 30 hommes, E101/650 n²6, E101/169 n 15.

124 Paiement du 20/04/1354: 35 dont 3 chevaliers, E101/169 $n^{\circ} 42$.

12528 hommes d'armes en Saintonge E101/168, 12, $n^{\circ} 88$. E101/169 $3 n^{\circ} 6$; voir aussi capra, Les bases sociales du pouvoir anglo-gascon, p. 280.

126 En Agenais, Périgord, Quercy et Limousin : Elie de Pommiers avec 29 hommes d'armes et 100 sergents et les Gontaud avec 20 hommes d'armes et 60 sergents, E101/169, 2,I, $n^{\circ} 67$ $(8 / 05 / 1354)$ et $\mathrm{E} 101 / 650 \mathrm{n}^{\circ} 71(16 / 11 / 1354)$.

127 Participe avec 40 personnes et 30 chevaux à l'expédition de 1359 en France, C61/72 mbr 2 ; contrat de guerre pour 40 hommes d'armes et 100 sergents à cheval E101/168, 12, n75. voir aussi garnison à Puynormand E372/199 fol. 39r, mbr 1-2; E403/374 mbr14; E101/168, 3, fol..11v; E101/650 n' 104 .

128 Capra, les bases sociales du pouvoir anglo gascon, pp. 281-282, n. 62-65 Raymond de Pellegrue (1354: E101/650 $n^{\circ} 72$, E101/168, 12, $n^{\circ} 80$; E101/169, 2,II $n^{\circ} 25$ et $35 ; 3 n^{\circ} 12$; E101/650 n133 et 149. contrats de Gaillard d'Agassac E101/168, $12 \mathrm{n}^{\circ} 7 ;$ E101/169, 2, I, $\mathrm{n}^{\circ}$ 45 et $51 ; \mathrm{E} 101 / 171,4, \mathrm{I}, \mathrm{n}^{\circ} 29 ; \mathrm{E} 101 / 171,4, \mathrm{II}, \mathrm{n}^{\circ} 26 ; \mathrm{E} 101 / 650 \mathrm{n}^{\circ} 34,91,101$. 
par G. Bois qui y voit une la «réaction d'une classe humiliée» ${ }^{129}$; leur cas semble démontrer que les circonstances ont offert des chances exceptionnelles à ceux qui ont survécu aux batailles et aux pestes. A côté des nobles ruinés, la Gascogne des années 13501360 connaît des cadets établis de façon inespérée qui auraient pu regarnir les rangs de leur milieu social, amenuisé par la concentration des fortunes, si la génération suivante avait connu des heures aussi fastes. Le cas des frères de Pommiers, bien qu'exceptionnel, suggère des vues moins négatives sur la noblesse, souligne sa capacité d'adaptation et l'esprit d'initiative de certains de ses membres.

Guillaume Sanche et Elie ont combattu avec des gages de banneret, leurs cadets végétèrent au service d'Edouard III comme chevaliers ou écuyers, tandis qu'un ou deux membres d'une branche cadette servaient le roi de France avec le même modeste rang, parmi une nuée d'autres hommes d'armes méridionaux de petit état, très rarement chevaliers et souvent d'une noblesse assez incertaine ${ }^{130}$. Il faudrait envisager une comparaison entre le pourcentage de chevaliers dans les troupes gasconnes des deux côtés, et il conviendrait de s'interroger sur les structures familiales de la noblesse méridionale, et sur sa désaffection pour la chevalerie vers 1360-70, quand les chevaliers de petite fortune étaient légion au temps des Recognitiones feodorum. De ce point de vue, les trois ou quatre chevaliers qui se rencontrent dans la fratrie des Pommiers vers 1360-1365 sont peut-être un cas atypique, lié à une réussite exceptionnelle.

\section{Service du Prince, influence sociale et fidélité}

Une partie d'entre eux, et singulièrement des chevaliers qui ont combattu sous les ordres d'Elie ou de Guillaume-Sanche, l'ont fait sans doute à plusieurs reprises; ils avaient là un vivier de relations, de protégés et pourquoi pas d'amis fidèles, qui leur donnait le réseau d'influence que la quasi absence d'arrière-vassaux dans le pays ne pouvait leur offrir, sur la base des relations féodales traditionnelles. De ces clients militaires des frères Pommiers, nous ne savons rien que par une mention de hasard, lorsqu'un protégé de Guillaume-Sanche, Fortin de Possignac fait prisonnier Robert de Beaujeu en Saintonge. ${ }^{131}$ En fait, ce que l'on saisit le mieux de la clientèle de Guillaume-Sanche et Elie ne tient pas à des affaires militaires, mais ecclésiastiques, et là encore l'avantage revient au cadet, certainement beaucoup plus entreprenant: le sire d'Arbanats intervient auprès du pape en faveur de clercs périgourdins, mais surtout bordelais, et pas seulement de familiers ou de proches parents. ${ }^{132}$

Dans l'érosion continue des patrimoines, le service du prince, les honneurs, les profits et l'influence qu'il apportait, purent paraître indispensables à maintenir son état, toujours est-il que le fils de Guillaume-Sanche III, Guillaume-Sanche IV, trahit en 1377 sur la promesse d'une pension -comme son feu oncle Elie — et d'une compagnie de deux cents hommes d'armes et cent arbalétriers-comme son père et son oncle-, bref tout ce que Edouard III et son fils avaient offert à la génération précédente, et que seul Charles $\mathrm{V}$

129 «Noblesse et crise des revenus seigneuriaux», La noblesse au Moyen Age, $X F^{e}-X V^{e}$ siècle. Essais à la mémoire de Robert Boutruche, réunis par P. Contamine, Paris , 1976, p. 228, et P. Contamine, Guerre, Etat et société à la fin du Moyen Age, Etudes sur les armées du roi de France, 1337-1494, Paris, 1972, p. 73.

130 P. Contamine, Ibid., p. $178,181$.

131 A. Huillard-Bréholles, Titres de la maison ducale de Bourbon, Paris, 1867, p. 120.

132 Suppliques à Urbain V, 3/03/1363, 3/06/1373, 17/11/1363, 9/12/1363 etc. 
pouvait désormais lui proposer. Un chef de guerre en touchant les gages de sa retenue, n'empochait-il que sa propre solde? D'une certaine façon Guillaume-Sanche IV tourne sa fidélité vers un état plus riche et évolué que le duché d'Aquitaine, et seul susceptible de lui fournir les fruits du pouvoir qui convienne à son rang. Les finances gasconnes et anglaises ne permettent plus vers 1370/1380, de maintenir la fidélité de la noblesse, sur les bases politiques qui avaient si bien réussi du temps d'Elie de Pommiers, la situation est même en train de se retourner contre le roi-duc. En 1375, Guillaume Sanche IV de Pommiers a pensé non sans de solides raisons que la seule sauvegarde possible consistait à rallier la cause de Charles V. ${ }^{133}$ On connait la mansuétude du gouvernement anglo-gascon en ce genre d'occasions. Or le vicomte de Fronsac fut impitoyablement frappé et décapité. On ne toléra pas qu'il fît ce que tant d'autres avaient couramment pratiqué. Le sénéchal Thomas de Felton qui avait une longue expérience des affaires gasconnes pour avoir fait connaissance avec ce pays dans la suite du Prince Noir vingt ans plus tôt ${ }^{134}$ ne pouvait que songer à la capacité d'entrainement du personnage ${ }^{135}$, or le vicomte n'avait encore pour cela d'autre qualité pour que d'être le fils de son père. $P$. Capra y dénotait la preuve que «la relative discrétion du seigneur de Pommiers (Guillaume-Sanche III) ne peut masquer qu'il est le chef moral des activistes militaires» et leur principal interprète auprès de l'administration..

Les Albret et les Grailly et Bertrand de Montferrand étaient aussi des seigneurs très importants, ils jouissaient auprès des vassaux aquitains du prestige qui s'attache à la considération qu'on nourrit pour les puissants, mais plutôt que parmi ces grands personnages, c'est chez un des leurs parvenu au rang de grand seigneur, que les nobles gascons ont préféré trouver leur inspiration et leur justification. Guillaume-Sanche de Pommiers, paré d'une réussite particulière sans que celle-ci l'ait par trop coupé de son milieu, a été certainement le meilleur représentant de ceux sur lesquels reposait si largement la fortune du duché, selon P. Capra. Mais nous n'avons bien sûr pas de preuve directe, faute de sources narratives un tant soit peu bavardes sur les goupes d'affinité et de choix politiques dans la noblesse gasconne vers 1355/1360

Cette appréciation globale de la fidélité nobiliaire concorde fortement avec les tendances historiographiques actuelles ${ }^{136}$, quant à la situation particulière de GuillaumeSanche III, au premier rang, sans être le tout premier, et surtout sans y être né, nous nous limiterons à l'envisager comme un cas, dont il resterait à voir s'il a des parallèles. Le dossier réuni par P. Capra était riche de beaucoup des interrogations actuelles quant aux effets de genèse de l'Etat moderne sur la noblesse et quant à la part active que ce milieu social y a joué.

Françoise Bériac/Eric Ruault

133 Bordeaux sous les rois d'Angleterre, sous la dir. d'Y. Renouard, Bordeaux, 1965, pp. 408-410.

134 Register of Edward the Black Prince, t. IV, 99, 161, 207, 229, 246, 361 etc.

135 E. Ruault, La famille de Pommiers, pp. 105-110.

136 P. Contamine (sous la dir. de), L'Etat et les aristocraties; M.-T. Caron, Noblesse et pouvoir royal. 